\title{
Planificación de un modelo integral de cuidados en una adolescente sometida a biopsia renal
}

\author{
Rita Rocío Márquez-Díaz \\ Hospital General Universitario Gregorio Marañón. Madrid. España
}

Como citar este artículo:

Márquez-Díaz RR. Planificación de un modelo integral de cuidados en una adolescente sometida a biopsia renal. Enferm Nefrol. 2019 0ct-Dic;22(4):436-44

\section{Resumen}

Descripción caso: adolescente de 14 años que presentaba hematuria y proteinuria aisladas desde hace apenas un año, en tratamiento farmacológico. Candidata para ser sometida a la técnica, ingresa en la unidad de nefrología pediátrica. En la valoración inicial, se detectó un posible caso de trastorno de la conducta alimentaria (TCA), puesto en conocimiento del equipo de salud.

Descripción del plan de cuidados: Se llevó a cabo un plan de cuidados integral que se centraba tanto en los aspectos físicos como psicosociales del individuo. En cuanto a los diagnósticos seleccionados, previo a la biopsia primó el temor y, tras ella, el deterioro de la integridad tisular y de estos a su vez derivaron los de disposición para mejorar los conocimientos, disposición para mejorar el afrontamiento, riesgo de sangrado, riesgo de infección, retención urinaria y dolor agudo.

Evaluación del plan: La paciente estuvo hospitalizada durante 48 horas, ya que después de la prueba presentó globo vesical. Durante su ingreso, quiso manejar su régimen terapéutico y conocer medidas para controlar el estrés ante la punción. Fue dada de alta con los problemas físicos resueltos y motivada para seguir aprendiendo sobre su proceso de enfermedad.

Conclusiones: A la vista de nuestros resultados podemos concluir que el plan de cuidados aplicado a esta paciente

\section{Correspondencia:}

Rita Rocío Márquez Díaz

E-mail: ritarocio.marquez@salud.madrid.org adolescente fue efectivo respecto a todos los resultados esperados (NOC).

PALABRAS CLAVE: biopsia; enfermedades renales; adolescente; enfermería holística; terminología normalizada de enfermería.

\section{Planning a comprehensive model of care in an adolescent undergoing renal biopsy}

\section{Abstract}

Case description: 14-year-old adolescent who presented hematuria and proteinuria isolated for just one year, in pharmacological treatment. Candidate to undergo the technique, she is admitted to the pediatric nephrology unit. In the initial assessment, a possible case of eating behavior disorder was detected, reported to the health team.

Description of the care plan: A comprehensive care plan focused on both the physical and psychosocial aspects of the individual was carried out. Regarding the selected diagnoses, prior to the biopsy, the fear prevailed and, after the procedure, the deterioration of the tissue integrity, and in turn, derived the diagnosis of willingness to improve knowledge, willingness to improve coping, risk of bleeding, risk of infection, urinary retention and acute pain.

Evaluation of the care plan: The patient was hospitalized for 48 hours, since after the procedure she presented a bladder balloon. During her admission, she wanted to manage her therapeutic regimen and learn 
about measures to control stress before puncture. She was discharged with the physical problems solved and motivated to continue learning about her disease process.

Conclusions: In view of our results we can conclude that the care plan applied to the adolescent patient was effective with respect to all the expected results (NOC).

KEYWORDS: biopsy; kidney diseases; adolescent; holistic nursing; standardized nursing terminology

\section{Introducción}

La biopsia renal (BR) consiste en una prueba diagnóstica que determina qué tipo de anomalía es la que está afectando al funcionamiento normal del riñón, vigente desde el año $1951^{1}$. La información recolectada del examen histológico del tejido renal puede ser útil en el diagnóstico, pronóstico y tratamiento de la enfermedad renal ${ }^{1}$.

Las patologías del riñón son detectadas a través de un análisis de orina, en el que puede aparecer microhematuria y proteinuria, es decir, pérdidas de pequeñas cantidades de sangre y proteínas en la orina, respectivamente ${ }^{2}$.

Aunque estos indicios sugieren la existencia de una enfermedad renal, no proporcionan un diagnóstico de certeza ${ }^{2}$. No obstante, la biopsia tiene ciertos riesgos por lo que antes de su realización se requiere, como mínimo3:

1) Agotar todos los procedimientos no invasivos.

2) Considerar que la información suministrada proporciona un diagnóstico de certeza.

3) Asegurar que se puede hacer estudio histológico completo.

4) Prever que el informe histológico influye en el tratamiento y pronóstico

A su vez, las indicaciones de este procedimiento son controvertidas dependiendo del país, del centro y de la opinión del clínico, y a su vez son diversas, encontrándose el síndrome nefrótico idiopático (siendo la primera indicación en España), las alteraciones urinarias asintomáticas como la hematuria y proteinuria, la insuficiencia renal aguda sin causa aparentemente clara y el síndrome nefrítico agudo en la etapa adulta ${ }^{3}$.
Sin embargo, antes de someterse a esta intervención, es necesario realizar un estudio preliminar sobre la situación clínica del paciente ya que existen contraindicaciones importantes como son las alteraciones de la coagulación, la hipertensión no controlada, la infección urinaria del espacio perirrenal o de la zona de punción, la anemia grave y la presencia de riñones pequeños (inferior a 10 $\mathrm{cm})$ o con anomalías anatómicas 4 .

La BR consiste en extraer dos cilindros renales con el propósito de ser examinados a posteriori por el servicio de anatomía patológica. La técnica que se usa en la práctica habitual es la percutánea ecodirigida en tiempo real ${ }^{1}$. Esto es, puncionar con una aguja a través de la piel uno de los riñones, seleccionando mediante ecografía dirigida, la zona más adecuada, bajo anestesia local ${ }^{5}$.

El paciente debe permanecer en posición de decúbito prono y colaborar con movimientos respiratorios6. Otras alternativas a la biopsia percutánea son: biopsia a cielo abierto, acceso transyugular, biopsia por laparoscopia 0 en posición supino-oblicua ${ }^{3}$.

Finalizada la intervención, es imprescindible mantener reposo absoluto durante 24 horas en decúbito supino, para así de esta manera evitar complicaciones post-biopsiab. Al ser una técnica invasiva siempre existe un cierto riesgo como puede ser la hemorragia, que si es macroscópica puede provocar un shock hipovolémico, la hipertensión arterial (riñón de Page), el hematoma perirrenal, el dolor, la infección, la punción de órganos cercanos y la fístula arteriovenosa ${ }^{6}$.

La labor de la enfermera es fundamental a la hora de realizar los cuidados antes, durante y después de la biopsia. Por esta razón, se ha elaborado un plan de cuidados de enfermería para unificar los criterios de actuación permitiendo detectar diagnósticos focalizados en el problema y de riesgo en el paciente sometido a $B R$, previniendo complicaciones y permitiendo así ofrecer una atención holística de los cuidados.

\section{Presentación del caso}

Adolescente de 14 años, que ingresa en la unidad de hospitalización de nefrología pediátrica para someterse a una BR percutánea. Presenta fractura traumática y acuñamiento de cuerpo vertebral de T7 con dorsalgia postraumática en diciembre de 2018. En cuanto a los antecedentes familiares, padre con diabetes mellitus y madre con hipertensión arterial, sus dos hermanos menores son 
sanos y no hay patologías renales conocidas en la familia. Hallazgo de albuminuria patológica y microhematuria con inmunoglobulina A (en adelante IgA) sérica elevada desde septiembre de 2018 en análisis repetidos. Inicialmente con disuria, sin mejoría clínica. Acudió a ginecología por este motivo y se realizaron pruebas de exudado y citología, ambas negativas. Ante esta situación fue derivada al servicio de Nefrología donde se está llevando a cabo su seguimiento, encontrándose a lo largo del mismo, asintomática, normotensa y sin edemas.

En los análisis de laboratorio, la función renal es normal pero las cifras de proteinuria y la hematuria presentan un discreto ascenso. Ante esto, se le pautó Lisinopril ${ }^{\circledR}$, $1 / 2$ comprimido $(2,5 \mathrm{mg})$ al acostarse, controlado por su madre. En las siguientes revisiones en la consulta médica, se optimizó la dosis del medicamento antes de plantear la prueba diagnóstica. Sara no toleró bien el fármaco, ya que le provocaba mareos el descenso de la tensión arterial producido por el aumento de la dosis de este $(5 \mathrm{mg})$. La proteinuria aislada era inferior a $1 \mathrm{~g} / 24$ horas, por lo que se planteó la $B R$, ya que, según el especialista, era una indicación antes de iniciar el tratamiento con esteroides. Posiblemente padeciese Nefropatía por IgA o también llamada enfermedad de Berger, causada porque el anticuerpo ( $\operatorname{IgA}$ ) se deposita en los riñones (7). Como factor de riesgo se detectó en su historia clínica varios episodios de amigdalitis bacteriana en la infancia. Tanto la paciente como sus padres aceptan la decisión de realizar la BR, ya que cumple con las premisas descritas anteriormente y no posee contraindicación alguna.

\section{Valoración enfermera}

En primer lugar, tras la exploración física, se valora el dato subjetivo de sus sentimientos en la etapa preoperatoria. Presenta miedo y, tal y como nos cuenta, tiene más temor a la canalización de la vía venosa periférica que a la propia intervención. También manifiesta ansiedad, debido a que, aunque le fueron resueltas todas las incertidumbres relativas a su estado de salud y a la biopsia, ahora no las recuerda.

En segundo lugar, se realiza una valoración de la paciente siguiendo el modelo de valoración de las 14 necesidades de Virginia Henderson (ver Tabla 1). Los datos son recogidos el día anterior a la realización de la BR a través de una entrevista personal con la paciente y sus padres.

Al ingreso, se realizaron por protocolo las siguientes escalas:
1. Norton Modificada: 20 puntos= paciente sin riesgo (dentro de la necesidad de alimentación).

2. Riesgo de caídas (J.H. Downton): 0 puntos= paciente $\sin$ riesgo.

Se le administró a la paciente el cuestionario MUST (MALNUTRITION UNIVERSAL SCREENING TOOL), para población general tras haber detectado en la evaluación un déficit en la ingesta o una ingesta inadecuada. En el test se obtuvo una puntuación de 3 puntos, riesgo alto de malnutrición. Debido a este resultado, se les pregunta a los padres sobre el estado nutricional de la paciente, comentándonos que tiende a saltarse las comidas o que solo toma alimentos "light" o "diet", comiéndolos muy despacio. En cuanto al IMC, Sara obtuvo 17,34 Kg/ $\mathrm{m}^{2}$, que, según la OMS, es bajo peso.

Estos signos nos hacen sospechar acerca de un trastorno de conducta alimentaria (TCA), por lo que se pusieron en conocimiento del pediatra responsable.

\section{Plan de cuidados}

Se detectó que la esfera psicosocial estaba gravemente comprometida en la etapa preoperatoria: la paciente se mostraba especialmente ansiosa al pensar en el dolor que suponía puncionarle y frustrada por no saber manejar su posible enfermedad, ya que se desentendía de la misma. $\mathrm{Y}$, tras la práctica de la $\mathrm{BR}$, se pudieron considerar como diagnósticos enfermeros de la paciente los que corresponden a "Deterioro de la Integridad Tisular", "Riesgo de sangrado", "Riesgo de infección", "Retención urinaria"y "Dolor agudo"8.

Por estas razones, se trataron aquellos diagnósticos de enfermería relacionados con la esfera física sin aminorar aquellos propios del ámbito psicosocial, resultando seleccionados los siguientes:

- "[00148] Temor relacionado con entorno desconocido y separación del sistema de apoyo y manifestado por impaciencia, tensión muscular, cambios en la respuesta fisiológica y focalización limitada a la fuente de temor" (véase Tabla 2) ${ }^{8}$.

La paciente se mostraba preocupada a la hora de tratar su cambio en su estado de salud. Constantemente preguntaba: "Enfermera, ¿me va a doler mucho?" Sentía inquietud sobre la venopunción, la biopsia y lo que iba a durar el proceso de su posible enfermedad, ya que ha- 
Tabla 1. Valoración de enfermería al ingreso según Necesidades de Virginia Henderson.

\begin{tabular}{|c|c|}
\hline Necesidad & Valoración \\
\hline Respiración & Respiración normal, ausencia de tos y ruidos respiratorios. Expectoración escasa. No fumadora. \\
\hline Alimentación & $\begin{array}{l}\text { Toma una dieta equilibrada moderada de sal, pero tiene problemas de apetito desde hace } 4 \text { meses. Ha perdido } 3 \text { kilos, pesando } \\
\text { actualmente } 47,2 \mathrm{~kg} \text {. No tiene problemas de masticación ni deglución. Su madre refiere que a veces la ha visto vomitar pero } \\
\text { la paciente lo justifica comentándole que últimamente le sienta mal la comida. } \\
\text { Observaciones: durante su estancia en el hospital la paciente solo ha comido la lechuga y las acelgas de la bandeja, refiriendo } \\
\text { que está inapetente por los nervios. Su padre cuenta que en casa apenas come y que si lo hace es sola en su propia habitación, } \\
\text { algo que atribuyen a la etapa de la adolescencia. En cuanto a la ingesta de líquidos bebe mucha agua, incluso más de } 2 \text { litros } \\
\text { al día. }\end{array}$ \\
\hline Eliminación & $\begin{array}{l}\text { Presenta proteinuria y hematuria diagnosticada desde el año 2018. De vez en cuando tiene episodios de diarrea/estreñimien- } \\
\text { to, actualmente ausentes. }\end{array}$ \\
\hline Movilización & Dedica cerca de 3 horas al día de ejercicio físico moderado. \\
\hline Reposo-Sueño & $\begin{array}{l}\text { La paciente refiere descansar "sin problemas". Duerme } 8 \text { horas diarias de lunes a viernes y alrededor de } 10 \text { horas los fines } \\
\text { de semana. Observaciones: a su madre le ha llamado la atención que en los últimos meses vista con ropa no ajustada, ya que } \\
\text { a siempre le ha disgustado dicho estilo. }\end{array}$ \\
\hline Vestirse/desvestirse & Autónoma. \\
\hline Temperatura & Sin alteración. \\
\hline $\begin{array}{l}\text { Higiene y estado de } \\
\text { la piel }\end{array}$ & Íntegra con color normal y sin lesiones en las mucosas. \\
\hline Seguridad & $\begin{array}{l}\text { Al ingreso ausencia de dolor (escala } E V A=0 \text { ). No existe riesgo de caídas. Consciente, orientada en espacio y tiempo. Alérgica } \\
\text { al Metamizol. Calendario vacunal completo. }\end{array}$ \\
\hline $\begin{array}{l}\text { Comunicación/ } \\
\text { relación }\end{array}$ & No hay dificultad para comunicarse. Porta gafas debido a la miopía. \\
\hline Creencias/religión & No existen conflictos entre las creencias y los cuidados de salud. \\
\hline Trabajo & Estudiante de Educación Secundaria Obligatoria. \\
\hline Ocio & Se entretiene viendo series en su móvil y usando redes sociales, a veces sale con sus amigas. \\
\hline \multirow{2}{*}{ Aprendizaje } & $\begin{array}{l}\text { Conoce el motivo por el que ha ingresado, aunque tiene dudas. Desconoce los cuidados asociados a su proceso, como, por } \\
\text { ejemplo, el régimen terapéutico. }\end{array}$ \\
\hline & $\begin{array}{l}\text { Observaciones: nos cuenta que al inicio mostró interés por tener más conocimiento sobre su estado de salud, pero después, } \\
\text { delegó la responsabilidad en su madre, puesto que es enfermera y ella es la que se ocupa de cuidarla. }\end{array}$ \\
\hline
\end{tabular}

bía pasado casi un año y no remontaban los parámetros analíticos.

En cuanto al procedimiento de la BR, se les explicaron a la paciente y a sus familiares la duración esperada del mismo, las rutinas preoperatorias (anestesia, dieta, pruebas/laboratorio, micción, preparación de la piel, terapia intravenosa; vestimenta, zona de espera para la familia y traslado al quirófano) y las posibles medidas de control del dolor.

Asimismo, del diagnóstico temor ${ }^{8}$, pudieron derivarse a su vez otros dos que están estrechamente enlazados:
- "[00161] Disposición para mejorar los conocimientos manifestado por expresar deseo de mejorar el aprendizaje" (véase Tabla 2) ${ }^{8}$

- "[00158] Disposición para mejorar el afrontamiento manifestado por expresar deseo de mejorar el manejo de los estresores" (véase Tabla 2) ${ }^{8}$

La razón por la que se planteó el primer diagnóstico fue que la paciente delegaba por completo en su madre, profesional sanitario, el control de su tratamiento. Desde que empezó a percibir un cambio en su estado de salud se mostraba apática, pero al verse hospitalizada sintió 
Tabla 2. Diagnósticos (NANDA), resultados (NOC) e intervenciones (NIC) enfermeras antes de la biopsia renal.

\begin{tabular}{|c|c|c|}
\hline NANDA & NOC & NIC \\
\hline $\begin{array}{l}\text { [00148] Temor } \\
\text { Factores relacionados: } \\
\text { - Entorno desconocido. } \\
\text { - Respuesta innata a estímulos. }\end{array}$ & $\begin{array}{l}\text { [1301] Adaptación del niño a la hospitali- } \\
\text { zación. } \\
\text { • [130101] Ansiedad. } \\
\text { Escala t Desde SIEMPRE demostrado } \\
\text { hasta NUNCA demostrado. } \\
\text { (P. Inicial } 2 \text { frecuentemente demostrado } \rightarrow \\
\text { P. Final } 4 \text { raramente demostrado). } \\
\text { - [130118] Cooperación en los procedi- } \\
\text { mientos. } \\
\text { - [130127] Mantiene el control. } \\
\text { Escala m Desde NUNCA demostrado hasta } \\
\text { SIEMPRE demostrado. } \\
\text { (P. Inicial } 1 \text { nunca demostrado } \rightarrow \text { P. Final } 3 \text { a } \\
\text { veces demostrado). }\end{array}$ & $\begin{array}{l}\text { [5820] Disminución de la ansiedad. } \\
\text { [5610] Enseñanza: prequirúrgica. }\end{array}$ \\
\hline $\begin{array}{l}\text { [00161] Disposición para mejorar los } \\
\text { conocimientos. } \\
\text { Características definitorias: } \\
\text { - Expresa deseo de mejorar el aprendizaje. }\end{array}$ & $\begin{array}{l}\text { [1209] Motivación } \\
\text { • [120913] Acepta las responsabilidades de } \\
\text { las acciones. } \\
\text { Escala m Desde NUNCA demostrado hasta } \\
\text { SIEMPRE demostrado. } \\
\text { (P. Inicial } 1 \text { nunca demostrado } \rightarrow \text { P. Final } 3 \\
\text { a veces demostrado). } \\
\text { [1813] Conocimiento: régimen terapéutico } \\
\text { - [181302] Responsabilidades de autocui- } \\
\text { dado para el tratamiento en curso. } \\
\text { - [181306] Régimen de medicación } \\
\text { prescrita. } \\
\text { Escala u Desde NINGÚN conocimiento } \\
\text { hasta Conocimiento EXTENSO. } \\
\text { (P. Inicial I ningún conocimiento } \rightarrow \text { P. Final } \\
3 \text { conocimiento moderado). }\end{array}$ & $\begin{array}{l}\text { [5540] Potenciación de la disposición de } \\
\text { aprendizaje. } \\
\text { [5614] Enseñanza: medicamentos prescritos. }\end{array}$ \\
\hline $\begin{array}{l}\text { [00158] Disposición para mejorar el afron- } \\
\text { tamiento } \\
\text { Características definitorias: } \\
\text { - Expresa deseo de mejorar el manejo de los } \\
\text { estresores. }\end{array}$ & $\begin{array}{l}\text { [1862] Conocimiento: manejo del estrés. } \\
\text { • [186215] Técnicas efectivas de disminu- } \\
\text { ción del estrés. } \\
\text { • [186213] Técnicas de meditación efecti- } \\
\text { vas. } \\
\text { Escala u Desde NINGÚN conocimiento hasta } \\
\text { Conocimiento EXTENSO (P. Inicial l ningún } \\
\text { conocimiento } \rightarrow \text { P. Final } 3 \text { conocimiento } \\
\text { moderado). }\end{array}$ & $\begin{array}{l}\text { [6040] Terapia de relajación. } \\
\text { [5880] Técnica de relajación. } \\
\text { [1460] Relajación muscular progresiva. }\end{array}$ \\
\hline
\end{tabular}

pesar por no haber participado antes y quiso hacerse responsable de su propia conducta.

De esta manera, se le proporcionó a la paciente información sobre las responsabilidades de autocuidado en cuanto al régimen de medicación prescrito.

Con respecto al segundo diagnóstico, la paciente no soportaba las agujas, pero ante esto, expresó deseo de mejorar el conocimiento sobre las estrategias de gestión del estrés. No estaba diagnosticada de belonefobia, que es el miedo extremo, incontrolable e irracional hacia las agujas, pero sí sentía el miedo como una emoción ${ }^{11}$.
Otro de los diagnósticos enfermeros valorados y que se centra en el ámbito físico fue el de deterioro de la integridad tisular. En este caso se enunció así:

- "[00044] Deterioro de la integridad tisular relacionado con procedimiento quirúrgico y manifestado por lesión tisular" (véase Tabla 3) 3 .

La biopsia es una técnica invasiva mediante la cual se extrae una pequeña muestra del tejido a estudiar, por lo que generará una lesión a consecuencia de elloº

Una de las complicaciones más temidas de la biopsia es la hemorragia. Cuando la hemorragia se vierte al espacio retroperitoneal, puede complicarse con disminución de la hemoglobina e hipovolemia. 
Tabla 3. Diagnósticos (NANDA), resultados (NOC) e intervenciones (NIC) enfermeras tras practicar la biopsia renal.

\begin{tabular}{|c|c|c|}
\hline NANDA & NOC & NIC \\
\hline $\begin{array}{l}\text { [00044] Deterioro de la integridad tisular } \\
\text { Factores relacionados: } \\
\text { - Procedimiento quirúrgico. }\end{array}$ & $\begin{array}{l}\text { [1102] Curación de la herida: por primera } \\
\text { intención. } \\
\text { • [110209] Edema perilesional. } \\
\text { • [110205] Secreción serosanguinolenta } \\
\text { de la herida. } \\
\text { Escala h Desde EXTENSO hasta NINGUNO } \\
\text { (P. Inicial } 1 \text { ninguno } \rightarrow \text { P. Final } 1 \text { ninguno). }\end{array}$ & $\begin{array}{l}\text { [3660] Cuidados de las heridas. } \\
\text { [3590] Vigilancia de la piel. }\end{array}$ \\
\hline $\begin{array}{l}\text { [00206] Riesgo de sangrado. } \\
\text { Factores de riesgo: } \\
\text { - Traumatismos. }\end{array}$ & $\begin{array}{l}\text { [0413] Severidad de la pérdida de sangre. } \\
\text { • [41313] Palidez de piel y mucosas } \\
\text { • [41316] Disminución de la hemoglobina. } \\
\text { Escala n Desde GRAVE hasta NINGUNO. } \\
\text { (P. Inicial } 1 \text { ninguno } \rightarrow \text { P. Final } 1 \text { ninguno). } \\
\text { - [41302] Hematuria. } \\
\text { Escala n Desde GRAVE hasta NINGUNo } \\
\text { (P. Inicial } 2 \text { leve } \rightarrow \text { P. Final } 2 \text { leve). }\end{array}$ & $\begin{array}{l}\text { [4010] Prevención de hemorragias. } \\
\text { [6680] Monitorización de los signos vitales. }\end{array}$ \\
\hline $\begin{array}{l}\text { [00004] Riesgo de infección. } \\
\text { Factores de riesgo: } \\
\text { - Procedimiento invasivo. }\end{array}$ & $\begin{array}{l}\text { [0703] Severidad de la infección. } \\
\quad \text { [070307] Fiebre. } \\
\quad \text { [070311] Malestar general. } \\
\text { Escala n Desde GRAVE hasta NINGUNO } \\
\text { (P. Inicial } 1 \text { ninguno } \rightarrow \text { P. Final } 1 \text { ninguno). }\end{array}$ & [6540] Control de infecciones. \\
\hline $\begin{array}{l}\text { [00023] Retención urinaria } \\
\text { Factores relacionados: } \\
\text { - Obstrucción del tracto urinario. }\end{array}$ & $\begin{array}{l}\text { [0503] Eliminación urinaria. } \\
\text { • [50301] Patrón de eliminación. } \\
\text { - [50313] Vacía la vejiga completamente. } \\
\text { Escala a Desde GRAVEMENTE comprometi- } \\
\text { do hasta N0 comprometido. } \\
\text { (P. Inicial } 3 \text { moderadamente comprometido } \rightarrow \\
\text { P. Final } 5 \text { no comprometido). }\end{array}$ & $\begin{array}{l}\text { [0620] Cuidados de la retención urinaria. } \\
\text { [0590] Manejo de la eliminación urinaria. }\end{array}$ \\
\hline $\begin{array}{l}\text { [00132] Dolor agudo } \\
\text { Factores relacionados: } \\
\text { - Agentes lesivos físicos (p. ej., absceso, am- } \\
\text { putación, quemadura, corte, levantar objetos } \\
\text { pesados, procesos quirúrgicos, traumatismos). }\end{array}$ & $\begin{array}{l}\text { [2102] Nivel del dolor. } \\
\text { - [210201] Dolor referido. } \\
\text { - [210223] Irritabilidad. } \\
\text { - [210224] Muecas de dolor. } \\
\text { Escala n Desde GRAVE hasta NINGUNO } \\
\text { (P. Inicial } 4 \text { leve } \rightarrow 5 \text { ninguno) }\end{array}$ & $\begin{array}{l}\text { [2210] Administración de analgésicos. } \\
\text { [1400] Manejo del dolor. } \\
\text { [6482] Manejo ambiental: confort. }\end{array}$ \\
\hline
\end{tabular}

Para evitar la hemorragia, es imprescindible que el paciente guarde reposo al menos 24 horas en posición de decúbito supino y beba abundantes líquidos. Hay que vigilar tanto la presión arterial como el pulso y supervisar la zona de punción y el color de la orina6.

Existen signos y síntomas que indican un sangrado interno, como son el aumento de la frecuencia cardíaca, la disminución de la tensión arterial, palidez de piel y mucosas, ansiedad, etc (6). Por esta razón, hay que controlar las constantes vitales, siendo registradas con cierta frecuencia: cada 30 minutos durante las primeras 3 horas y después cada hora.

- "[00004] Riesgo de infección manifestado por procedimiento invasivo" (Tabla 2$)^{8}$.
La infección también puede provenir de la inserción y mantenimiento del catéter venoso periférico. A menudo esta causa no se tiene en consideración o se considera como problema menor. No obstante, se ha constatado que las vías periféricas son causa de bacteriemia nosocomial $^{2}$.

Tras la práctica de la biopsia, la se recuperó en la planta de hospitalización. No hubo incidencias en las primeras horas, pero terminando la mañana, la paciente presentaba distensión abdominal debido a la presencia de globo vesical. En la ecografía se observó que, efectivamente, tenía bastante residuo posmiccional. Ante esto, se colocó sondaje vesical con el cual evacuó una gran cantidad de líquido. 
Por último, mencionar el diagnóstico enfermero de "[00132] Dolor agudo" (véase Tabla 3) ${ }^{8}$, ya que en la etapa postoperatoria debe tenerse en cuenta como $5^{\text {a }}$ constante vital ${ }^{12}$. Precisamente porque el dolor es, junto con los signos derivados de irritación peritoneal y la hipotensión, el síntoma guía más importante de la presencia de complicaciones derivadas de la biopsia ${ }^{6}$.

\section{Evaluación del Plan}

La evolución en la planta tras la BR fue progresiva. Al principio la paciente refirió un dolor en la zona de punción medido en escala EVA de 4. Se le administró el analgésico pautado, Paracetamol, y solo precisó 2 dosis a lo largo de todo su ingreso, esto es 48 horas, yéndose de alta con una puntuación de 0 en dicha escala. La herida presentaba buen aspecto, sin signos de infección ni sangrado. Sí que presentó leve hematuria, contemplada como complicación menor de la BR. Se les informó a los padres y a la paciente que este signo era normal en los primeros días, pero que, posteriormente iría remitiendo, por lo que había que ir vigilándolo en el domicilio. Con referencia al globo vesical, desapareció por completo al sondar a la paciente, retirándosela al día siguiente sin inconvenientes. En cuanto a la ansiedad manifestada frente a la hospitalización, las estrategias de relajación, autocontrol y enseñanza ayudaron a que esta disminuyese, llegando al punto de que Sara a veces colaborase en los procedimientos. De esta forma, se facilitó su estancia en el hospital. Por último, la paciente nos demostró interés por aprender sobre su régimen terapéutico. Las puntuaciones de los indicadores ("responsabilidades de autocuidado para el tratamiento en curso" y "régimen de medicación prescrita") evolucionaron de 1 : ningún conocimiento a 2: conocimiento escaso, debido a que no fue posible explicarle en un día con profundidad los detalles de su actual condición. No obstante, se le transmitió nuestra disponibilidad para establecer un ambiente de aprendizaje lo antes posible, a la vez que se le proporcionaron fuentes acreditadas de información. Sin la motivación de la paciente, el sostén de la familia, los cuidados integrales de la enfermería y el equipo interdisciplinar no habría sido posible un óptimo progreso durante su ingreso.

Hay que tener en cuenta que en la valoración inicial se detectó una sospecha de un TCA, identificado por los síntomas y signos de alarma descritos: adelgazamiento, vómitos, ejercicio excesivo, descenso del peso, cambios en el patrón de la alimentación (dietas hipocalóricas) y la vestimenta (ropa holgada). Ante esto, se avisó al pediatra responsable, el cual solicitó una petición de interconsulta a los servicios de Digestivo, Endocrinología y Psiquiatría.

\section{Discusión}

Tras la BR, hay que vigilar estrechamente la posible aparición de complicaciones. Por ende, el paciente debe permanecer al menos 24 horas en el hospital, tras lo cual puede ser dado de alta si no han aparecido complicaciones. La hemorragia es considerada la más grave mientras que la más frecuente es la microhematuria, que cede a lo largo de los días siguientes ${ }^{4}$. La justificación de la severidad de la hemorragia es que puede provocar, por un lado, un shock hipovolémico en el paciente por la cantidad de sangre perdida ${ }^{2}$. Y, por otro lado, si la sangre se almacena en la cápsula renal, da lugar a un taponamiento renal que cursa con hipertensión por estímulo del sistema renina-angiotensina (riñón de Page)². Esta prueba diagnóstica es un procedimiento seguro con un bajo riesgo de complicaciones, aunque hay que prestar atención a las mismas tras su realización ${ }^{2}$. Es aquí donde el personal de Enfermería adquiere un papel importante en la monitorización y observación clínica.

Otro punto que tratar es la hospitalización en los niños y adolescentes. Es bien conocido el hecho de que un ingreso puede afectar al bienestar y desarrollo de un niño/adolescente, pero también puede ser una oportunidad para enriquecer su experiencia y lograr que desarrolle resiliencia, entendida esta como la capacidad de un individuo para adaptarse positivamente a situaciones adversas ${ }^{13,14}$. En efecto, la paciente se enfrentó a situaciones de estrés, como pudo ser la venopunción o la canalización periférica, y salió fortalecida. Desde este punto de vista, la participación del niño/adolescente, la familia y el equipo de salud son importantes en la creación de un ambiente acogedor dentro de la institución ${ }^{15}$.

El proceso enfermero debe centrarse en la respuesta del paciente-familia a los problemas de salud ${ }^{16}$. Con la realización de este plan de cuidados se ha podido observar que no solo los profesionales se han de basar en las necesidades físicas de la persona sino también tener en cuenta las psicosociales, ya que influyen en el bienestar del paciente si no son cubiertas ${ }^{17}$. Atender al paciente en su totalidad, animarle a que participe en su recuperación, ayudarle a encontrar respuestas, aclarar dudas, enseñarles y apoyarles psicológicamente son algunas de las actividades que forman parte de la planificación de un modelo integral de cuidados ${ }^{17}$, mediante el cual se pudo identificar precozmente un posible caso de TCA en la pa- 
ciente. Según la literatura, el despistaje temprano de los trastornos de la alimentación se asocia con un aumento de las probabilidades de una evolución favorable ${ }^{18}$. EI pronóstico empeora conforma más tiempo pase entre el inicio del diagnóstico y el tratamiento ${ }^{18}$.

La escasa concienciación de los profesionales ante esta problemática y la falta de tiempo por la gran presión asistencial impiden profundizar en la atención integral de los pacientes ${ }^{18}$. Esto hace imprescindible la formación de diversos profesionales, tanto del ámbito sanitario como docente, respecto a los TCA ${ }^{18}$. Dentro de este aprendizaje se engloban los signos y síntomas de alarma, los factores predisponentes/precipitantes y las características y graves consecuencias de este tipo de trastornos ${ }^{19}$.

A la vista de nuestros resultados podemos concluir que el plan de cuidados aplicado a esta paciente adolescente fue efectivo respecto a todos los resultados esperados (NOC).

Por último y no por ello menos importante, existen pequeñas aportaciones científicas de casos clínicos con pacientes sometidos a BR abordados desde una perspectiva integral, por ello, sería interesante desarrollar líneas futuras de investigación que indaguen en el conjunto de las necesidades del paciente (y la familia) y los cuidados holísticos que han de ofrecerse como respuesta ${ }^{16}$.

Recibido: 18-09-19

Revisado: 25-09-19

Modificado: 28-09-19

Aceptado: 30-09-19

\section{Bibliografía}

1. Hernández FR. Biopsia renal. NefroPlus. 2009; 2(1):1-8.

2. Bandari J, FullerTW, Turner RM, D'Agostino LA. Renal biopsy for medical renal disease: Indications and contraindications. Can J Urol 2016;23(1):8121-6.

3. Fiorentino $M$, Bolignano $D$, Tesar $V$, Pisano A, Van Biesen W, D'Arrigo G, et al. Renal biopsy in 2015 - From epidemiology to evidence-based indications. Am J Nephrol 2016;43(1):1-19.
4. Santangelo L, Netti GS, Giordano P, Carbone V, Martino $M$, Torres DD, et al. Indications and results of renal biopsy in children: a 36-year experience. World J Pediatr 2018;14(2):127-33.

5. Hama T, Nakanishi K, Shima Y, Mukaiyama H, Togawa $H$, Tanaka $R$, et al. Renal biopsy criterion in children with asymptomatic constant isolated proteinuria. Nephrol Dial Transplant 2012;27(8):318690.

6. Zhu MS, Chen JZ, Xu AP. Factors that can minimize bleeding complications after renal biopsy. Int Urol Nephrol 2014;46(10):1969-75.

7. Coppo R. How early renal biopsy has to be performed in children with isolated asymptomatic proteinuria? Nephrol Dial Transplant 2012;27(8):3016-7.

8. International N. Diagnósticos Enfermeros: Definiciones y Clasificación. 2018-2020. 1la Edición. Barcelona: Elsevier; 2018.

9. Morrhead S, Swanson E, Johnson M, Mass ML. Clasificación de Resultados de Enfermería (NOC). $6 a$ Edición. Barcelona: Elsevier; 2018.

10. Butcher HK, Bulechek GM, Dochterman JM, Wagner CM. Clasificación de Intervenciones de Enfermería (NIC). 7a Edición. Barcelona: Elsevier; 2018.

11. McLenon J, Rogers MAM. The fear of needles: A systematic review and meta-analysis. J Adv Nurs. 2019;75(1):30-42.

12. Leyva Carmona M, Torres Luna $R$, Ortiz San Román L, Marsinyach Ros I, Navarro Marchena L, Mangudo Paredes $A B$, et al. Documento de posicionamiento del Grupo Español para el Estudio del Dolor Pediátrico (GEEDP) de la Asociación Española de Pediatría sobre el registro del dolor como quinta constante. An Pediatría2019;91(1):1-7.

13. Jedeloo S, van Staa AL, Latour JM, van Exel NJA. Preferences for health care and self-management among Dutch adolescents with chronic conditions: A Q-methodological investigation. Int J Nurs Stud 2010;47(5):593-603.

14. Ullán AM, Serrano I, Badía M, Delgado J. Hospitales amigables para adolescentes: Preferencias de los pacientes. Enferm Clin. 2010;20(6):341-8. 
15. Ullán AM, Belver MH, Serrano I, Delgado J, Badía M. Perspectives of Youths and Adults Improve the Care of Hospitalized Adolescents in Spain. J Pediatr Heal Care. 2012;26(3):182-92.

16. Potter PJ, Frisch N. Holistic Assessment and Care: Presence in the Process. Nurs Clin North Am. 2007;42(2):213-28.

17. McEvoy L, Duffy A. Holistic practice - A concept analysis. Nurse Educ Pract [Internet]. 2008;8(6):412-9.

18. Álvarez-Malé ML, Castaño IB, Majem LS. Prevalencia de los trastornos de la conducta alimentaria en adolescentes de Gran Canaria. Nutr Hosp. 2015;31(5):2283-8.

19. Barajas Iglesias B, Jáuregui Lobera I, Laporta Herrero I, Santed Germán MÁ. Eating disorders during the adolescence: Personality characteristics associated with anorexia and bulimia nervosa. Nutr Hosp. 2017;34(5):1178-84.

Este artículo se distribuye bajo una Licencia Creative Commons Atribución-NoComercial 4.0 Internacional. https://creativecommons.org/licenses/by-nc/4.0/

\section{Open Access (c) ()}

